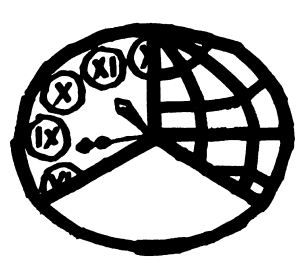

\title{
HISTORIA DE LAS ANTIGUAS INVESTIGACIONES REALIZADAS EN EL YACIMIENTO DE LA CUEVA DE AMBROSIO (VÉLEZ-BLANCO, ALMERÍA, ESPAÑA)
}

\author{
History of the ancient excavations at La Cueva de Ambrosio \\ (Vélez-Blanco, Almería, Spain)
}

Sergio Ripoll López ${ }^{1}$, Francisco J. Muñoz Ibáñez ${ }^{1}$ y Jesús F. Jordá Pardo ${ }^{1}$

Recibido el 4 de julio de 2013. Aceptado el 16 de julio de 2013

Resumen. La importancia del yacimiento de La Cueva de Ambrosio (Vélez-Blanco, Almería, España) queda reflejada en las numerosas excavaciones que en él se han llevado a cabo. Ya a principios de siglo H. Breuil y F. de Motos realizaron una campaña que proporcionó la primera punta de muesca de retoque abrupto del levante español. Este tipo de útil fue dado a conocer internacionalmente en la famosa publicación de H. Breuil sobre las subdivisiones del Paleolítico superior. Una de las efemérides que celebramos en este Congreso Internacional es precisamente el centenario de esta primera excavación.

Palabras clave: historiografía, Paleolítico superior, Andalucía, sureste español.

Abstract. The importance of the site of La Cueva de Ambrosio (Vélez-Blanco, Almería, Spain) is reflected in the numerous excavations that have taken him out. By the beginning of the century H. Breuil and F. Motos made a campaign that provided the first notch tip abrupt retouch the Spanish Levant. In this International Congress we celebrate the centenary of this first excavation.

Keywords: historiography, Upper Palaeolithic, Andalusia, Southeast of Spain.

\section{INTRODUCCIÓN}

Es en el pueblo de Vélez-Blanco (Almería, España), donde se inicia la historia de la investigación de La Cueva de Ambrosio, en la persona de Don Federico de Motos, farmacéutico, erudito y gran aficionado a la arqueología. Sus contactos con el Marqués de Cerralbo, con el profesor Hernández-Pacheco, con Juan Cabré y otros investigadores de la época, le llevaron a entrar en contacto con el abate Henri Breuil. Este, en 1911, estuvo en la comarca velezana para calcar y copiar las pinturas de los abrigos de la Cueva de los Letreros, de la Fuente de los Molinos y otros existentes en el macizo del Maimón y en los alrededores de Vélez-Blanco. El primero de ellos fue dado a conocer por M. de Góngora y Martínez en su libro Antigüedades Prehistóricas de Anda- lucía que se publicó en 1868 y constituye uno de los libros pioneros en el conocimiento del arte prehistórico.

Precisamente la noticia de como el abate Breuil llegó a Vélez-Blanco la recogemos en un discurso pronunciado en la inauguración de la IV sesión del Congreso Internacional de Ciencias Prehistóricas y Protohistóricas, celebrado en Madrid en 1954 (Breuil 1956):

... nous descendimes a faire une visite de quelques jours à Herrerías, Cuevas de Vera (?) (Almería), où le grand archéologue belge Louis Siret nous reçut. Le magnifique explorateur de tout le pays alentour et fondateur de la science préhistorique recente de début du Néolithique à l'arrivée des Carthaginois, vivait comme un ermite, entre sa mine et ses restes

(') Departamento de Prehistoria y Arqueología. Facultad de Geografia e Historia. Universidad Nacional de Educación a Distancia. Ciudad Universitaria.Paseo Senda del Rey 7. E-28040 Madrid (España).sripoll@geo.uned.es·fmunoz@geo.uned.es·jjorda@geo.uned.es. 
antiques. Grace a lui nous parvinimes jusqu'à VélezBlanco, tout au nord de cette province sub-desertique, pour y visiter "Los Letreros de Góngora et plusieurs autres sites qui nous a signalé le très distingué pharmacien du lieu, don Federico de Motos, doue d'un veritable sens et d'une vraie passion pour l'archéologie. Son amitié et le vive charme de ses relations furent une conquête, et, grâce à son agréable et precieux concours, j'eu plusieurs années de nouvelles roches peintes à relever alentour; ...

La primera noticia que se tiene de La Cueva de Ambrosio, aparece en una carta de fecha 24 de Noviembre de 1911, que F. de Motos dirige al abate Breuil, en la que el erudito explica los resultados de una pequeña trinchera que mandó hacer en una cueva cuyo relleno le parece paleolítico (Fig. 1). En otra carta fechada el 13 de junio de 1912, F. de Motos contesta a $\mathrm{H}$. Breuil sobre las mejores épocas para realizar la excavación que empezaba a perfilarse.

En 1912 el abate Breuil publicó en el Congrés International d'Anthropologie et Archéologie Préhistoriques celebrado en Ginebra (Suiza), su obra: Les Subdivisions $d u$ Paléolithique Supérieur et leur signification. En esta publicación el abate presenta una punta de muesca típica de Cueva de Ambrosio (Fig. 2), con el pedúnculo ligeramente curvado, que le había entregado F. de Motos para su estudio, procedente posiblemente de una recogida superficial o bien de la pequeña trinchera abierta a finales de 1911, con el fin de averiguar si el yacimiento contenía restos arqueológicos y al periodo al que correspondía.

A finales de 1912, concretamente el 17 de diciembre, Motos escribe a $\mathrm{H}$. Breuil para expresarle su deseo de iniciar los trabajos en la cueva. La excavación que realizaron $\mathrm{H}$. Breuil y F. de Motos, según la correspondencia dirigida por el segundo al primero no se inicia hasta mediados de abril de 1913 como podemos deducir de la carta de 10 de abril de 1913 en la que el erudito velezano se muestra de acuerdo con las fechas que propone el abate Breuil. Se prevé su llegada a Vélez-Blanco el día 15 de abril a través de la estación de Lorca. Los resultados de esta excavación fueron dados a conocer en una breve nota que Breuil publica en Les Travaux executés de l'année 1913 (Breuil 1913).

Grâce au concours de notre disntingué ami don Federico de Motos, nous avons pu commencer les travaux d'exploration dans le gisement de la Cueva de Ambrosio, à 20 kilomètres environ au nord de Velez Blanco. Nous avons surtout préparé les fouilles ultérieures, en enlevant une grande partie des blocs effondrés sur le gisement dans sa partie d'amont; nous avons, non sans difficulté, retruvé de ce côté, sur une large surface, des assises archéologiques en place. Elles nous ont livré, dans la faible partie que nous avons fouillé, une grande quantité de silex d'aspect paléolithique supérieur, et quelques os travaillés très simples; la faune se compose de Cerf, de Bouquetin, de Capridé plus petit, de Cheval et soutout d'une immense quantité d'ossements de Lapins. Ce fait rapproche de ceux analogues constatés dans la region de Valence, dénote que le lapin a tenu une très grande place dans l'alimentation des peuplades de l'extrême fin du quaternaire de ces regions.

De esta nota podemos deducir que la excavación la realizaron posiblemente en la zona oeste y en el interior del abrigo, donde los estratos paleolíticos están bastante altos, mientras que en el exterior, estos mismos estratos, en la actualidad, están a casi $2 \mathrm{~m}$ de profundidad (en el interior es difícil saber donde estaba el suelo original, aunque suponemos que debía estar a unos $6 \mathrm{~m}$ de profundidad con respecto al nivel actual en la zona oeste). Es muy posible que no excavaran los niveles paleolíticos propiamente dichos, mucho más ricos, sino la zona de contacto entre el nivel de grandes bloques entre los que aparecen restos epipaleolíticos y el nivel de tierras amarillentas con algunas piezas solutrenses. Los materiales de esta excavación estuvieron depositados en el Institut de Paleontologie Humaine de Paris, hasta que en el año 1973, gracias a las gestiones de los profesores M. Almagro Basch y M. Almagro Gorbea, entraron a formar parte, junto con los materiales de la Cueva del Castillo (Puente Viesgo, Santander), de las colecciones del Museo Arqueológico Nacional.

Otra parte de los materiales, posiblemente los recogidos en superficie, o en la pequeña trinchera realizada en 1911 por F. de Motos, junto con la punta de muesca, en silex melado, permanecieron en la colección Motos, quien, en 1930 la vendió al Servicio de Investigaciones Prehistóricas (S.I.P.) de Valencia para ser integrados en el entonces naciente museo de esa institución. Estos materiales fueron recogidos por el profesor Luis Pericot, que el 15 de mayo de 1930 se personó en Vélez-Blanco, donde tuvo la oportunidad de estudiar la tan traída y llevada punta de muesca, sin saber que algunas semanas más tarde, él las iba a encontrar en la cueva del Parpalló (Gandía, Valencia). La colección procedente de La Cueva de Ambrosio, conservada en el S.I.P. consta de 178 piezas líticas, 5 fragmentos de restos malacológicos y un sólo resto óseo y fue publicada por J. Alcacer Grau en 1972 (Fig. 3).

De la venta de su colección, F. de Motos, sólo conservó la punta de muesca como recuerdo, la cual fue entregada al centro anteriormente citado por su viuda doña Caridad Torrecilla al morir él en el mes de septiembre de 1932.

Otra parte de la colección fue vendida al Museo de Ciencias Naturales y al Museo Antropológico de Madrid, y en 1942, pasó a formar parte de las colecciones del Museo Arqueológico Nacional de Madrid. Puestos en contacto con 

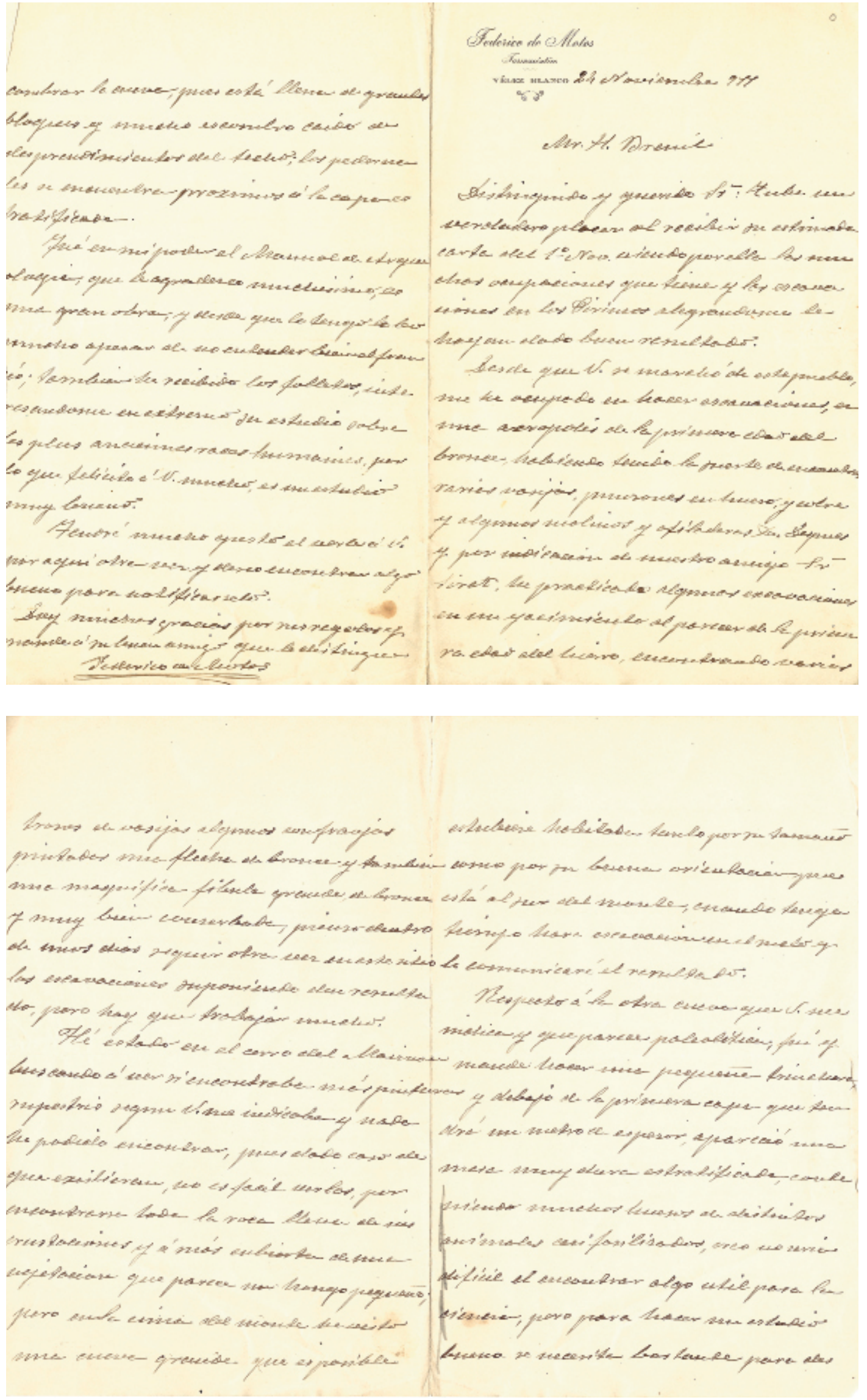

$\Delta$ Figura 1 a y b. Copia de la carta dirigida por Don Federico de Motos al abate Henri Breuil con fecha 24 de noviembre de 1911 en la que le comunica el descubrimiento de una gran cavidad con abundantes restos presumiblemente paleolíticos. 

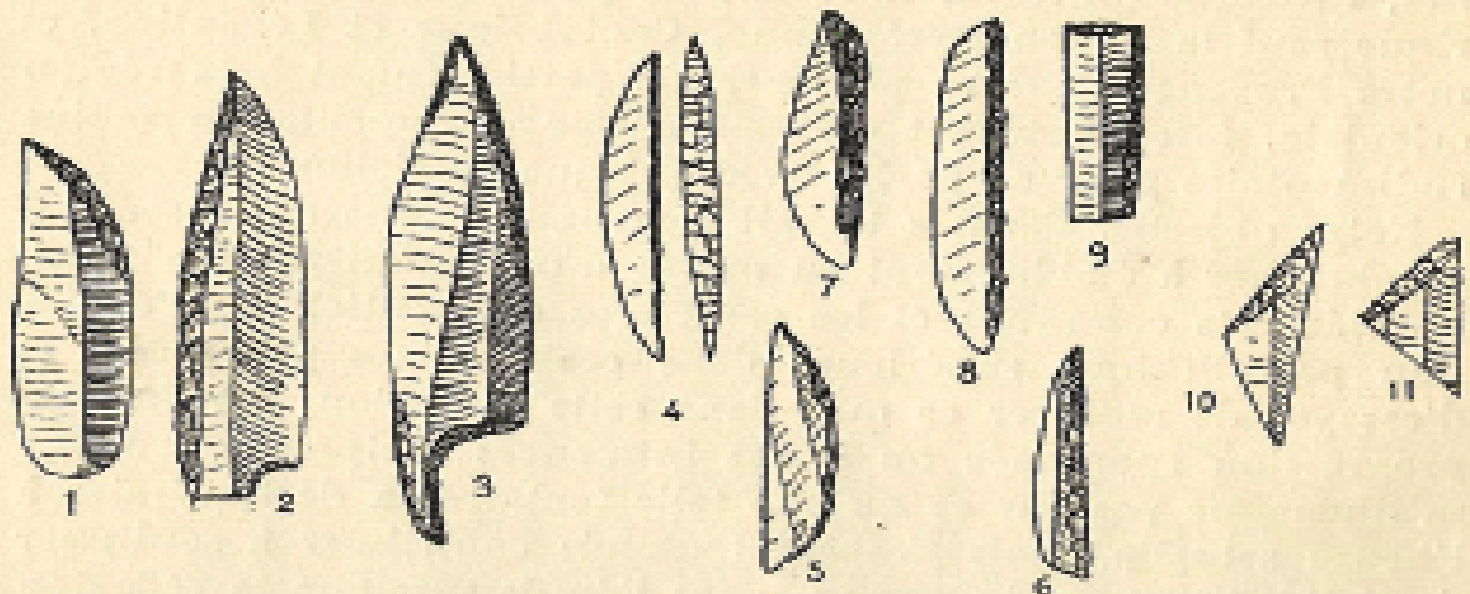

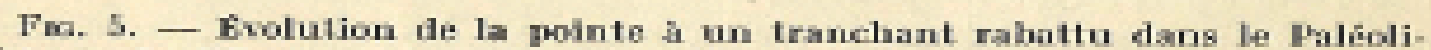

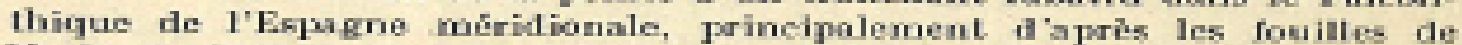

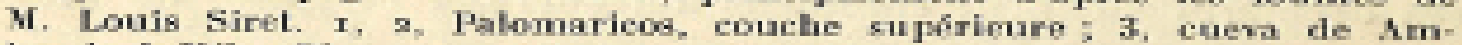

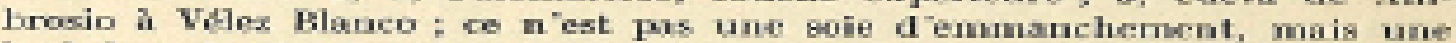
barbelure lasilaine qui prolonge la pitere vers lo las en forme de palon-

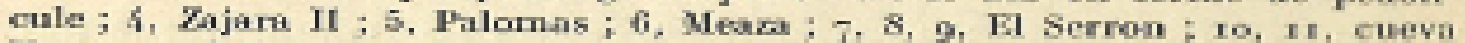
Humion. Fehrile is

\ FiguRA 2. Copia de la lámina publicada por el abate Henri Breuil en su obra Les Subdivisions du Paléolithique Supérieur et leur signification en la que figura una punta de muesca hallada en La Cueva de Ambrosio (Breuil 1913).

SERVICIO DE INVESTIGACION PREHISTORICA DipUtACION PROVINCIAL de VALENCIA SERIE DE TRABAJOS VARIOS Nom. ${ }^{4} 3$

\section{A T A L O G O}

COLECCION FEDERICO DE MOTOS

EN EL MUSEO DE PREHISTORIA DE VALENCIA

$$
\begin{aligned}
& \text { JOSE ALCACER GRAU } \\
& \text { Prologo del } \\
& \text { Dr. D. LUIS PERICOT GARCIA }
\end{aligned}
$$

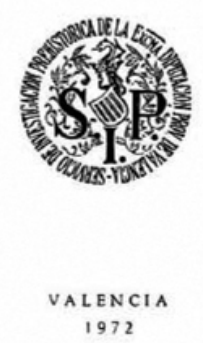

ム Figura 3. Portada del Catálogo de la colección Federico de Motos en la serie del Servicio de Investigaciones Prehistóricas de la Diputación Provincial de Valencia (Alcacer Grau 1972), en el que se recoge el inventario y estudio de todas aquellas piezas que la viuda del farmacéutico de Vélez-Blanco vendió al S.I.P. los herederos de F. de Motos, no hemos podido conseguir ningún tipo de documento, como cartas o escritos originales, en los que se diera alguna información, ya que todos sus papeles fueron vendidos al peso al morir el erudito velezano.

Las noticias sobre si Luis Siret excavó en La Cueva de Ambrosio, son bastante contradictorias. En su obra inédita L'Espagne Préhistorique, no menciona la excavación de Cueva de Ambrosio, sin embargo en una nota encontrada en unas cajas con materiales en el Museo Arqueológico Nacional, procedentes de las excavaciones de L. Siret en Almería, habla de la Cueva del Tesoro y dice:

Se halla situada en el Arroyo del Moral, a tres leguas al Norte de Vélez-Blanco (Almería).

Nosotros pensamos, después de haber visto el escaso material que se conserva en este museo de Madrid, compuesto fundamentalmente por piezas retocadas y prácticamente ningún resto de talla, que se trata de una recogida superficial o un obsequio de Breuil o Motos al arqueólogo belga. Hay que señalar también que el aspecto de los útiles de la colección Siret es muy parecido al que nosotros hemos estudiado de Ambrosio.

En algunos manuscritos conservados en el Museo Arqueológico Nacional, Luis Siret, hace referencia al yacimiento de Ambrosio, e incluso el propio Jiménez Navarro comenta en su publicación, la realización de una cata en la cueva por Siret, según noticia que le había transmitido Juan 
Cuadrado Ruiz, gran amigo e íntimo colaborador del insigne investigador belga.

En 1944, el profesor J. Martínez Santa-Olalla, estudió la posibilidad de emprender una serie de campañas de excavación en La Cueva de Ambrosio. Encargó estos trabajos al profesor E. Jiménez Navarro (Fig. 4), que en 1944, y acompañado durante unos días por el entonces Comisario Provincial de excavaciones de Almería, Don Juan Cuadrado Ruiz, empezó las excavaciones en la zona oeste del abrigo, en una zona intacta entre la pared de la cueva y un bloque de gran tamaño que divide el abrigo en dos. La primera capa de unos $10 \mathrm{~cm}$ había sido revuelta por clandestinos y no ofrecía ninguna garantía. A partir de la misma, en la tierra negruzca, propia de esta capa del yacimiento, se señala la existencia de bolsadas de ceniza con algún trozo de carbón "delimitadas por tres piedras de tamaño mediano"; revueltos con la ceniza aparecian con frecuencia "pequeños bolos de caliza o de arenisca esponjosa, con la superficie quemada y descompuesta como si hubiesen servido para apagar el fuego del hogar". También eran muy abundantes los huesos de animales y restos de comida. Así mismo, se hallaron instrumentos líticos y fragmentos cerámicos. De los primeros destaca Jiménez Navarro, su tosquedad, y de los segundos su riqueza decorativa. Aparecieron también objetos de adorno, y fragmentados, algunos huesos humanos.

En este primer nivel de Neolítico medio, según su excavador, entre el material lítico abundan los sílex, aunque escasean los microlitos y faltan totalmente las puntas de flecha. Los microlitos, que son las piezas mejor trabajadas de todo el conjunto, contrastan por su cuidada talla y finura con el resto de la industria. En este mismo nivel se encontraron varios fragmentos de hachas pulimentadas en anfibolita, arenisca y en esquisto, así como un alisador y un posible afilador. Se recogieron también varios machacadores de ocre y piedras de moler cereal.

Los hallazgos cerámicos forman el conjunto más interesante de la excavación de Jiménez Navarro en el yacimiento. Precisamente este nivel neolítico comienza en las capas más profundas con "cerámica lisa, gruesa y de poco tamaño, que generalmente adopta la forma de un cuenco hemisférico" y sigue a lo largo de todo el nivel alternando con la decorada a base de incisiones e impresiones. Es frecuente que esta cerámica aparezca impregnada de pintura roja en su superficie exterior y en algunos casos en el interior. A $45 \mathrm{~cm}$ de profundidad aparecieron dos vasijas enteras de barro fino, sin decorar; las dos estaban llenas de tierra negruzca con algún resto de carbón de pino.

Los útiles sobre hueso son escasos y se reducen a algunos punzones y varias puntas de asta de ciervo con marcas de pulimento. Entre los adornos existen varios brazaletes y algunos fragmentos de Pecten y Columbella perforados así como lo que tal vez podría ser una falange de animal, según

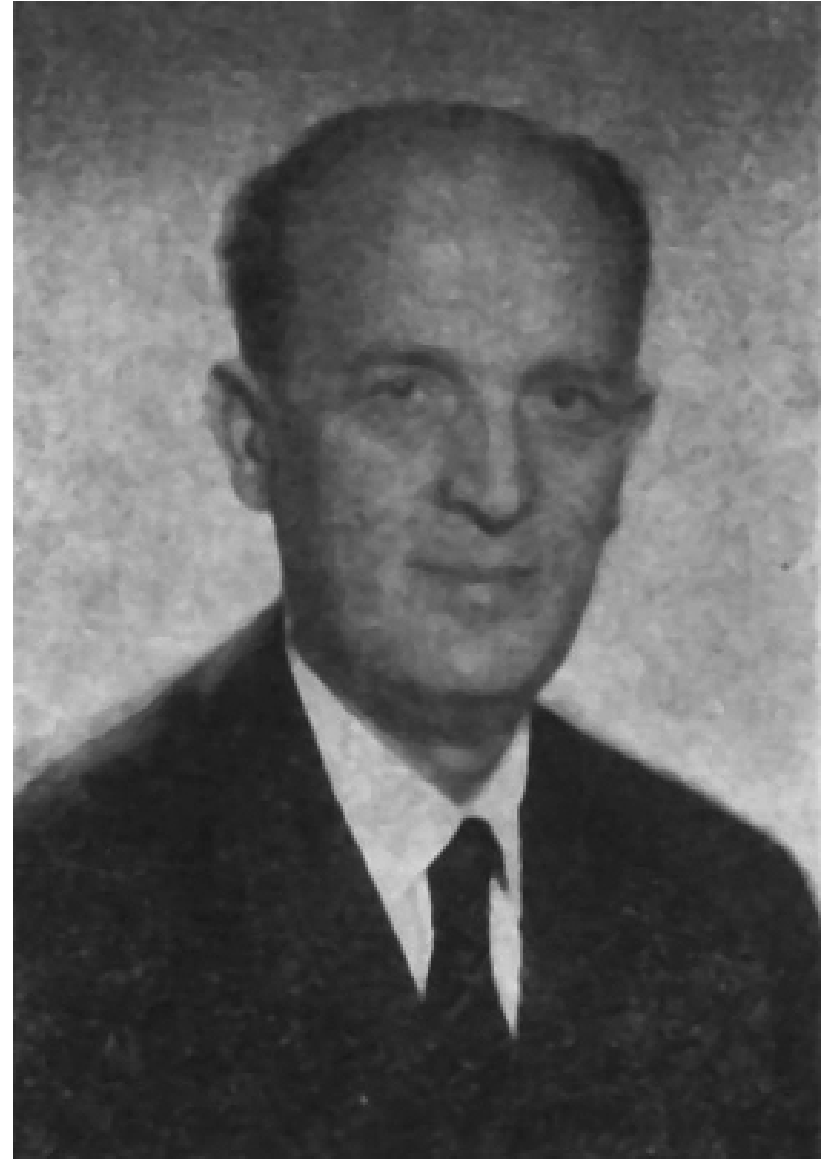

$\Delta$ Figura 4. Retrato de Ernesto Jiménez Navarro que excavó en La Cueva de Ambrosio en 1947.

se observa en el dibujo de la publicación, con un agujero transversal.

Hacia el metro de profundidad cambia el aspecto de la capa neolítica, se produce un empobrecimiento de los tipos cerámicos y la ausencia tanto de huesos trabajados como de objetos de adorno. Este nivel inferior -desgraciadamente mezcla del nivel Neolítico y otro Paleolítico, que bien pudiera ser Epipaleolítico o Magdaleniense, se caracteriza por el predominio de los tipos microlíticos, alguna cerámica primitiva, y toscos cuchillos de cuarcita. A estas piezas, cuchillos $u$ hojas retocadas, Jiménez Navarro las definía como hendidores [sic].

Estas excavaciones pusieron de manifiesto la existencia, en La Cueva de Ambrosio, de un rico estrato Neolítico que en algunos puntos alcanza los $2 \mathrm{~m}$ de potencia. Este nivel es muy representativo de la Cultura de las Cuevas y se corresponde con un Neolítico puro, pues faltan el metal y las puntas de flecha. Así mismo Jiménez Navarro señaló por primera vez la presencia aquí de la cultura del vaso campaniforme, atestiguada por la presencia de varios fragmentos.

E. Jiménez Navarro, no realizó otras campañas publicando dos notas (Jiménez Navarro 1962) que son los que nos sirven para explicar sus excavaciones. Clarisa Millán (Millán 1950) anotó, años después, la importancia de esta excava- 
ción y sus materiales tan interesantes para el estudio del Neolítico puro en el sureste. J. San Valero (San Valero 1946) tomó en consideración la importancia de estos materiales y S. Navarrete Enciso (Navarrete Enciso 1976) los relaciona en su tesis doctoral sobre la Cultura de las Cuevas en el sureste español.

\section{LAS CAMPAÑAS DE EXCAVACIONES DE E. RIPOLL PERELLÓ ENTRE LOS AÑOS 1958 Y 1964}

A través de la correspondencia mantenida entre el abate $\mathrm{H}$. Breuil y el profesor E. Ripoll Perelló, se aprecia la insistencia del primero para que se volvieran a iniciar los trabajos de excavación y estudio de Cueva de Ambrosio. En 1958, el Servicio de Investigaciones Arqueológicas de la Diputación Provincial de Barcelona a través del que entonces era su director, el profesor Luis Pericot, y en la ayuda económica de la Wenner-Gren Foundation for Anthropological Research de Nueva York, encargó al profesor E. Ripoll, el inicio de una serie de campañas en el yacimiento de la Cueva de Ambrosio.

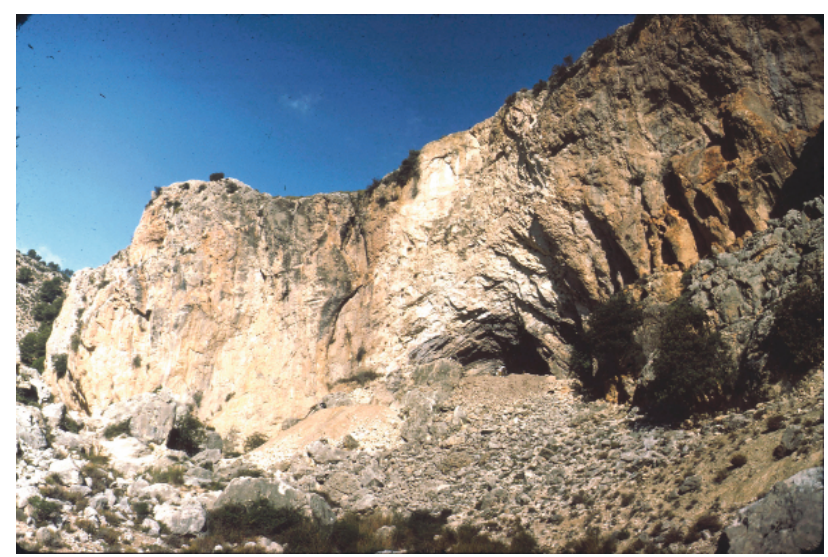

$\Delta$ Figura 5. Vista de La Cueva de Ambrosio tal como se encontraba en el año 1958 cuando el profesor Eduardo Ripoll Perelló inició las excavaciones.

En el mes de Junio de 1958 se realizó una pequeña campaña de prospección, para conocer el estado de conservación del yacimiento (Fig. 5), e intentar localizar otros en las cercanías del abrigo (Cueva Alta, Lavaderos de Tello, etc.). También se aprovechó para hacer una revisión de los calcos realizados por el abate $\mathrm{H}$. Breuil en la cercana Cueva de los Letreros y en La Fuente de los Molinos que ya fueron publicadas por este autor en su obra Les Peintures Rupestres Schématiques de la Peninsule lbérique en el año 1935. El 17 de octubre se procedió a solicitar el permiso de excavación que fue concedido por la Dirección General de Bellas Artes el 15 de noviembre de 1958. A continuación pasamos a explicar las campañas de excavación basándonos en los cuadernos de campo que $\mathrm{E}$. Ripoll puso a nuestra disposición.
La primera campaña de excavaciones se realizó desde el 19 de noviembre al 2 de diciembre de 1958 (Ripoll Perelló 1960-1961). Los trabajos se iniciaron limpiando una amplia zona de excavación, retirando gran parte de los bloques que cubrian el yacimiento. A continuación se limpió el primer nivel de tierras revueltas y llenas de excrementos de cabras y ovejas, ya que el abrigo como ya hemos explicado, se utilizaba desde siempre como paridera o refugio para los rebaños de la cortijada de La Cueva de Ambrosio.

Los trabajos de excavación propiamente dichos se empezaron abriendo dos pequeñas trincheras, a ambos lados del abrigo, es decir en los lados este y oeste del mismo para intentar averiguar donde se encontraban los niveles intactos. En la zona oeste, debajo de la tierra con excrementos, aparecía un sedimento removido casi en su totalidad, excepto en una pequeña zona de la misma que podía estar intacta.

En este lugar se profundizó hasta unos $0,70 \mathrm{~m}, \mathrm{mo}-$ mento en que afloró una tierra amarillenta, con grandes bloques. Esta capa tenía una potencia de 0,50 m, y buzaba fuertemente hacia el interior del abrigo. El material que se encontró en esta capa era bastante escaso. En la zona este del abrigo se limpió el sector de bloques de piedra caídos, y se empezó a quitar el revuelto, apareciendo, a menor profundidad la capa amarillenta sobre la que se hallaron algunos útiles. La zona de contacto entre esta capa y la siguiente, estaba bien diferenciada por una ligera capa de hogares en la zona oeste. Sin embargo en la zona este, esta zona de contacto estaba caracterizada por unas tierras finas con abundantes cantos de aristas vivas y de color amarillomarrón, en el que aparecía muy poco material.

En el sector oeste, la siguiente capa, era un potente nivel de unos 0,40 $\mathrm{m}$ de hogares de color muy negro. Aparecian muy pocas piezas de sílex y algunas piezas dentarias de grandes vertebrados. Debajo de la capa de hogares, salía un nivel muy espeso, 0,80 m de grandes piedras de derrumbamiento. Inmediatamente después existía una capa de tierra con restos aislados de hogares de unos 0,65 $\mathrm{m}$. Los hallazgos se componian sobre todo de raederas, raspadores de grandes dimensiones y bastantes hojas.

Al final de la campaña, no se profundizó más ya que se pensaba en la posible existencia de un nivel de Paleolítico medio y ya no quedaba mucho tiempo para excavarlo. En el sector oeste se descubrió una oquedad o concavidad interior, de unos $6 \mathrm{~m}$ de ancho por $12 \mathrm{~m}$ de largo y unos 0,60 $\mathrm{m}$ de altura, que había quedado libre de tierra al estar tapada su entrada por una brecha, en la que todavía hoy se pueden ver algunos materiales paleolíticos. Esta brecha se pudo haber formado con el agua que se filtra a través de una grieta situada encima de este punto. Se tapó la entrada para que no se pudieran meter animales y se silenció el hallazgo para poder encontrarlo intacto en la siguiente campaña.

La campaña del año 1960 se llevó a cabo durante el mes de noviembre (Ripoll Perelló 1960-1961, 1962). Se iniciaron los 
trabajos limpiando la zona de la trinchera abierta en el sector oeste, sacando las grandes piedras que se habian desprendido del techo y de los cortes, así como la tierra con excrementos de animales que habian vuelto a entrar en el yacimiento. Se abrieron también dos accesos al pequeño covacho interior. En la zona este del abrigo aparecieron bastantes restos cerámicos, entre ellos unos fragmentos de vaso cilíndrico con dos asas de mamelón y un fragmento de orza de grandes dimensiones con asas y decorada con incisiones. Los trabajos de excavación se empezaron en el ángulo sureste de la trinchera abierta en la campaña anterior, en la que se empezó a excavar el primer nivel que cubría los estratos solutrenses.

A la vez se iniciaron unos trabajos en la cueva superior 0 Cueva del Valle, en la que se abrió un trinchera para comprobar si contenía materiales arqueológicos. Durante el primer día de excavación sólo se encontraron algunos fragmentos muy dispersos.

Al mismo tiempo se empezó la excavación del covacho interior del yacimiento principal, quitando la capa gris que cubria casi todo el suelo. Aparecía poca cerámica y algunas piezas de sílex. Debajo surgía el primer estrato de tierra amarilla arcillosa, mezclada con muchas piedras, que se excavó y cribó muy cuidadosamente, apareciendo algunos restos líticos entre los grandes bloques. Debajo de este nivel, en la zona de contacto entre esta capa anterior y los niveles solutrenses, salió una capa de unos $10 \mathrm{~cm}$ de espesor, totalmente estéril, mezclada con algunas plaquetas, que a pesar de tener algunas manchas de ocre, no formaban ninguna figura. Inmediatamente aparecieron las tierras negras de los hogares en los que salen centenares de piezas de sílex y restos óseos, así mismo aparecieron algunas plaquetas. Durante esta campaña, en el fondo del covacho, se llegó hasta una capa muy fina de tierras arcillosas de color rojizo, posiblemente por la acción del fuego. Esta campaña concluyó volviendo a tapar las entradas al llamado fondo del covacho.

En el mes de febrero de 1961 y dado el gran interés que tenían estos materiales encontrados durante las campañas de 1958 y 1960, el Servicio de Investigaciones Arqueológicas, dirigido en ese momento por E. Ripoll, montó una exposición monográfica en el Museo Arqueológico Provincial de Barcelona para darlos a conocer al público.

La campaña de 1962 se realizó en el mes de junio, iniciándose la excavación con la apertura de una trinchera, para comunicar la escombrera con las zonas excavadas en las campañas anteriores, así como con una zona que se llamó A1 que se excavó durante esta campaña. Igualmente se abrió en la zona media del abrigo, bajo unos grandes bloques, la otra zona de excavación que se llamó A2. En ambas zonas se rebajó el terreno al mismo tiempo, excavando los restos del nivel neolítico y el nivel de grandes piedras y tierra amarillenta que se cribó con gran meticulosidad, apareciendo abundantes restos líticos y algunos caracoles (Theodoxus fluviatilis) con perforación intencional. A con- tinuación, después de la tierra amarilla, empezaron a salir numerosas capas de hogares con abundantes hallazgos.

A partir de este momento se marcó una cuadrícula de 2,70 m de lado que se subdividió en cuatro sectores, que se excavaron muy cuidadosamente, situando en planos de dispersión de cada nivel, todas las piezas de silex, el resto de talla, restos óseos, plaquetas con ocre, etc. En la zona inferior de este nivel aparecieron unas arenas casi estériles, - parece como si el Solutrense se empobreciese de arriba a abajo- debajo de estas arenas, la tierra se hacia más fina y rojiza, con abundantes materiales, para rarificarse rápidamente en un nivel de gravas estériles, de muy probable aportación hidráulica, que indica una etapa en la que el Arroyo del Moral era más caudaloso o discurría más alto, o ambas cosas a la vez. Al llegar a este nivel, se dio por finalizada la campaña.

De la campaña de 1963, puesto que fue el objeto de la memoria de licenciatura de uno de los firmantes, sólo diremos que se desarrolló en el mes de octubre y se excavaron dos zonas: la zona B que constituyó el objeto del estudio antes citado y que nos remitimos a la publicación del mismo (Ripoll López 1986) y el llamado fondo del covacho. En esta última zona se continuaron los trabajos de excavación descubriendo un nivel de tierras gris-verdosas con algunos útiles y debajo del mismo apareció lo que parecía ser la roca del abrigo en un gran estado de descomposición. Cuando se llegó a este punto en la zona del fondo del covacho, se dio por finalizada la excavación dadas las adversas condiciones climáticas existentes.

La campaña de 1964 tuvo lugar a principios del mes de octubre. Se iniciaron los trabajos en la zona del fondo del covacho, excavando una parte que quedaba bastante alta entre esta área y las zonas A1 y A2. La tierra que apareció en el primer nivel era gredosa, muy compacta y con escaso material. Debajo apareció el nivel de gravas, estéril, de unos $15 \mathrm{~cm}$ de espesor. La capa que apareció a continuación era de greda amarillenta y presentaba abundantes inclusiones de cenizas y carbones. En este nivel aparecieron agrupados, tres grandes tritones (Charonia lampas) junto con una abundante industria lítica.

Durante esta campaña participó en la excavación, durante unos dias, un equipo francés compuesto por el matrimonio F. Bordes y D. de Sonneville-Bordes y J. Tixier (Fig. 6), que excavaron unos pequeños cuadros en el lado izquierdo de la trinchera de acceso al covacho (Figs. 7 y 8). En esta zona debajo del nivel amarillo había otro de limos arcillosos en el que se encontraron una serie de piedras que parecian estar dispuestas de un modo intencional (¿estructura?) (Fig. 9). En este nivel de gredas amarillas, el matrimonio Bordes y Tixier encontraron abundantes restos solutrenses. En una limpieza que se efectuó en la zona Este del abrigo, se encontró una pequeña trinchera rellena de tierra gris superficial y piedras, posiblemente de las excavaciones realizadas 
por E. Jiménez Navarro en 1944. La campaña se cerró el 20 de octubre dejando todos los cortes limpios para, en la siguiente campaña, intentar localizar aquellos niveles de $\mathrm{Pa}$ leolítico medio que se suponían estaban debajo. La trinchera de acceso al covacho, tenía 4,80 m en la zona más profunda.

Durante dicha campaña de 1964 se tomaron cinco muestras para poder realizar dataciones radiocarbónicas, que se enviaron a la Universidad del Estado de Washington. Desgraciadamente, las dataciones, que sugerian que el Solutrense con puntas pedunculadas de este yacimiento se dataría entre 12.000 y 6.000 años antes de nuestra era, no son coherentes las unas con las otras. Sin duda debió de haber habido una contaminación de las muestras. Por otra parte también se cogieron muestras por D. de SonnevilleBordes, para la realización de un análisis sedimentológico que presentamos en la monografía sobre las excavaciones de 1963 (Ripoll López 1986).

En el mismo año 1964 se cursó una solicitud para que fuera concedida una subvención mayor, a fin de colocar unas vigas de cemento bastante recias, para calzar los bloques que estaban en suspensión en el interior del abrigo, ya que al sacar la tierra, se vio que estaban desgajadas del abrigo, y los trabajos de excavación en la zona resultaban extremadamente peligrosos. Esta subvención no fue concedida nunca y el permiso de excavación no fue renovado.

\section{LAS CAMPAÑAS POSTERIORES}

A partir de ese momento, la cueva quedó a merced de los excavadores clandestinos, hasta que, en 1975, M. Botella llevó a cabo una campaña de excavaciones. En efecto, en el mes de agosto de 1975 los componentes de un equipo del Laboratorio de Antropología de la Facultad de Medicina de la Universidad de Granada, dirigidos por M. Botella, realiza-

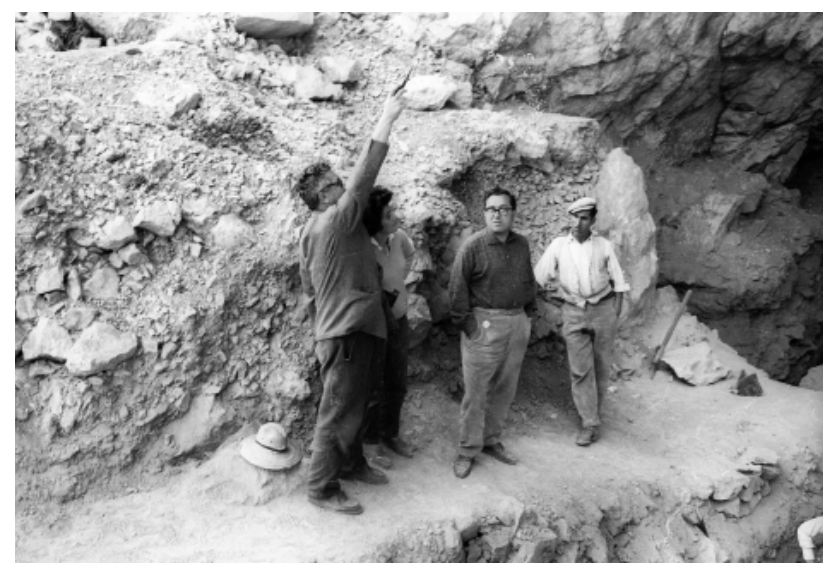

A Figura 6. Durante la campaña de 1964 participaron varios investigadores franceses como el matrimonio François Bordes y Denise de Sonneville Bordes y Jacques Tixier entre otros. En la foto junto a Eduardo Ripoll y el capataz Salvador Torrente. ron una pequeña campaña de excavación para comprobar si la cueva conservaba todavía algún nivel intacto. El yacimiento estaba muy destruido a causa de las acciones de los buscadores clandestinos. Se plantearon una serie de cortes. El primero de ellos, denominado corte exterior, se realizó en la zona este, fuera del abrigo, pero estaba muy revuelto y el hallazgo de grandes piedras les decidió a no seguir en él.

El denominado primer corte fue de 3 por $2 \mathrm{~m}$, y se hizo también en la zona este del abrigo, pero en el interior. De él se excavaron hasta $3 \mathrm{~m}$ de profundidad, después de haberlo subdividido en cuadriculas de 0,50 m de lado. Este corte también resultó estéril, ya que se trataba de un pellizco de falla, constituido por margas muy duras de color verdoso. El segundo corte se planteó en la zona oeste del abrigo con unas dimensiones de 1 por 1 metro en el que se diferenciaron 7 niveles epipaleolíticos. Este hallazgo de Epipaleolítico en una potencia de $2 \mathrm{~m}$ resulta sorprendente si se tiene en cuenta que en esta misma zona, según se sitúa el corte en la planta, nosotros realizamos el sondeo en el año 1986 y localizamos el II nivel con materiales solutrenses a unos $40 \mathrm{~cm}$ de la superficie, debajo de una acumulación de sedimentos totalmente revueltos y un nivel de arcillas prácticamente estéril. Extraña pues pensar que a pesar de las remociones de los clandestinos, no

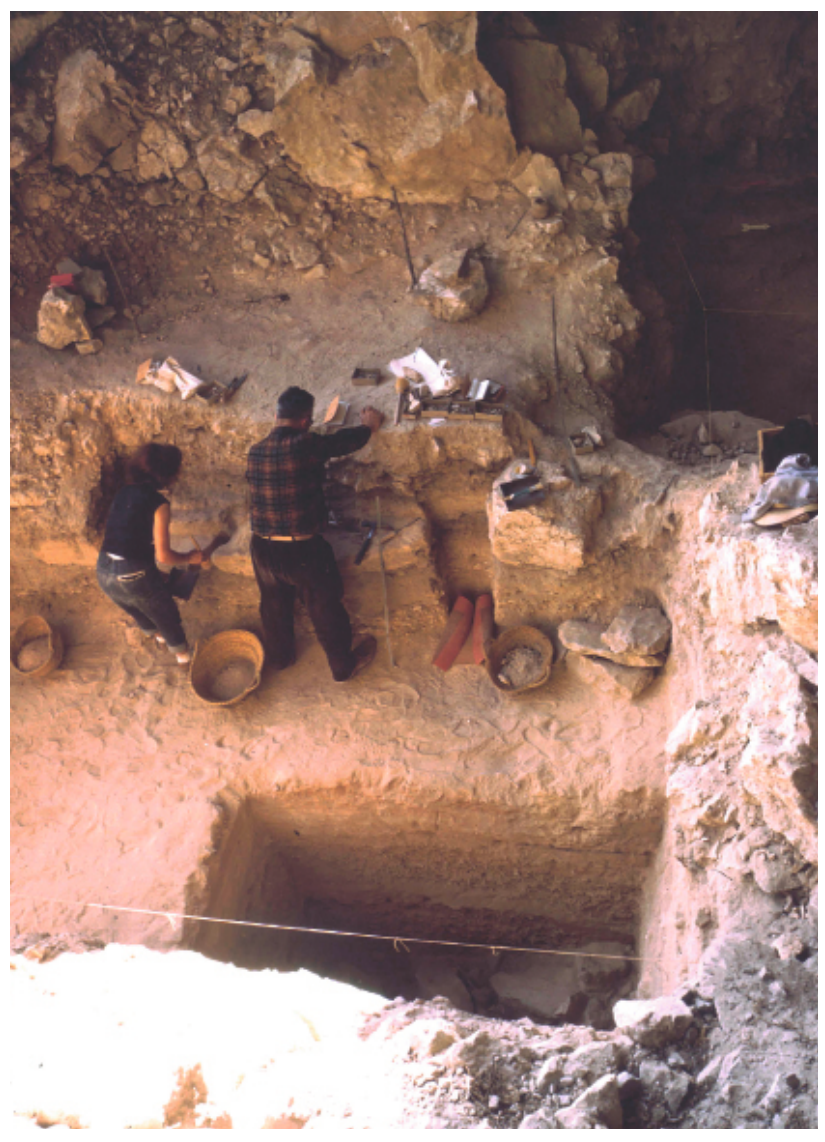

$\Delta$ Figura 7. Vista de la zona donde se excavó durante la campaña de 1964 en la que participaron algunos investigadores franceses. 


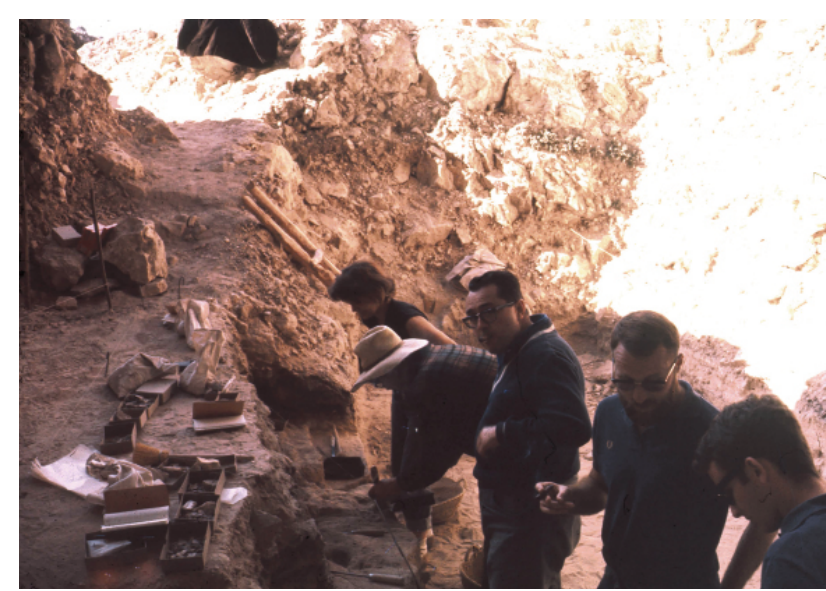

A Figura 8. El equipo de investigación hispano francés durante las tareas de excavación.

alcanzasen el estrato Solutrense habiendo profundizado $2 \mathrm{~m}$, en los que suponemos sería sedimento intacto. Justo delante del gran bloque que divide la cueva en dos partes, se cuadriculó otro corte de 3 por $2 \mathrm{~m}$, el corte tercero, que también resultó arqueológicamente estéril. El corte cuarto se realizó en el lado oeste del abrigo, en una superficie de 1 por $1 \mathrm{~m}$ y alcanzó una profundidad $2 \mathrm{~m}$, en la que diferenciaron 6 niveles en los que se encontraron materiales del Paleolítico superior.

Los materiales procedentes de esta excavación, a pesar de su pobreza, dieron como resultado una memoria de licenciatura, redactada por Ángela Suárez (Suárez 1980). En este trabajo se exponía que los niveles epipaleolíticos se debían incluir dentro de facies microlaminar de Fortea y presentaban unos paralelos muy claros con otros yacimientos del Mediterráneo español, como Les Mallaetes.

Por estas fechas se apuntó también, en una comunicación presentada por M. Botella en el Congreso Arqueológico Nacional de Vitoria de 1975 (comunicación oral no publicada), la existencia de un nivel auriñaciense en la base del relleno, pero la extrema pobreza de los materiales hallados, hizo que el propio investigador nos lo desmintiera personalmente en fecha posterior.

A mediados del año 1980, a instancias del Director del Museo Arqueológico Provincial Luis Siret de Almería, Ángel Pérez Casas, y del Ayuntamiento de Vélez-Blanco, se iniciaron los trabajos de cerramiento del abrigo mediante un muro de encofrado de $2 \mathrm{~m}$ de altura, con el fin de proteger el yacimiento que pudiera conservarse todavía. Este cerramiento finalizó el día 23 de diciembre de 1980.

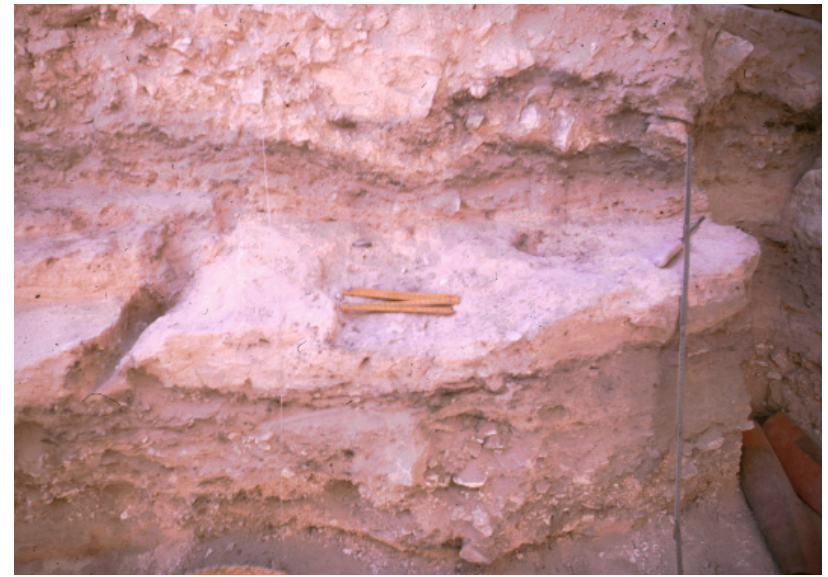

A Figura 9. Detalle del "hogar en cola" descubierto durante la campaña de excavaciones del año 1964.

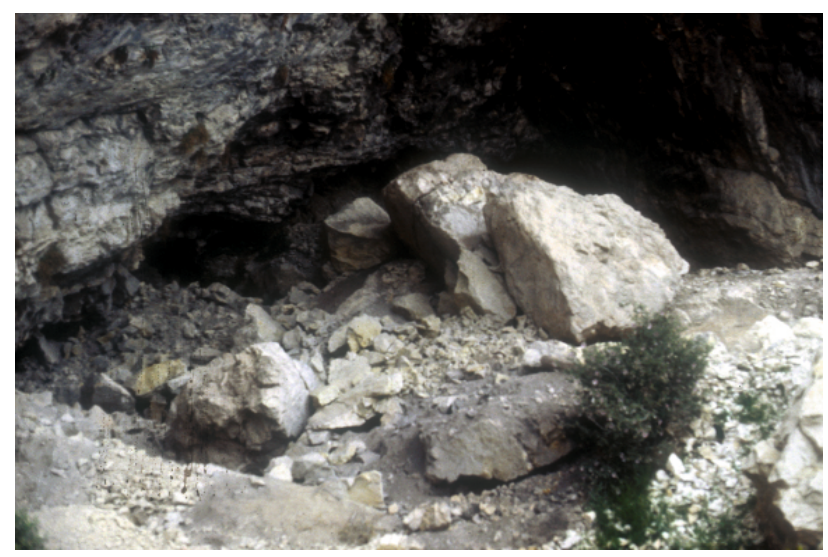

$\Delta$ Figura 10. Estado en que se encontraba el yacimiento en el año 1982 cuando uno de los firmantes (SRL) se hizo cargo de las campañas de excavación sistemática en La Cueva de Ambrosio.

En una visita que realizamos por estas fechas al abrigo, nos pareció muy interesante pedir un permiso de excavación, ya que el volver a iniciar los trabajos en el yacimiento, nos permitiría corroborar y completar algunas de las hipótesis deducidas a partir de los materiales extraídos en las campañas de excavación de E. Ripoll. Por este motivo solicitamos de la Subdirección General de Arqueología y Etnografía de la Dirección General de Bellas Artes y Archivos y Museos del Ministerio de Cultura, el correspondiente permiso y subvención, que nos fueron concedidos en 1982 (Fig. 10). El relato de las actuaciones que se llevaron a cabo a partir de entonces se explicita en otro texto de este mismo volumen. • 


\section{BIBLIOGRAFÍA}

Alcacer Grau, J. 1972: Catálogo de la colección Federico de Motos en el Museo de Prehistoria de Valencia. Trabajos Varios 43. Servicio de Investigaciones Prehistóricas. Diputación Provincial de Valencia. Valencia.

BREUIL, H. 1913: "Les subdivisions du Paléolithique Supérieur et leur signification". Congrés International d'Anthropologie et d'Archéologie Préhistoriques, Compte rendu de la XIVéme session, Genève 1912. Genève: 165-238 (reeditado en 1937).

- 1956: "La Préhistoire de l'Espagne". Congreso International de Ciencias Prehistóricas y Protohistóricas. Actas de la IV sesión. Madrid: 10-22.

Jiménez Navarro, E. 1962: "Excavaciones en Cueva de Ambrosio (Almería)". Noticiario Arqueológico Hispánico V (1956-1961): 13-48.
Ripoll López, S. 1986: El Solutrense de La Cueva de Ambrosio (VélezBlanco, Almería).Campaña de excavaciones de 1963. Excavaciones Arqueológicas en España 148. Ministerio de Cultura. Madrid.

Ripoll Pereló, E. 1960-1961: "Excavaciones en Cueva de Ambrosio (Vélez-Blanco, Almeria). Campañas de 1958-1960". Ampurias 2223: 31-44.

- 1962: "Excavaciones en Cueva de Ambrosio (Vélez-Blanco, Almería)". VII Congreso Arqueológico Nacional, Barcelona 1961. Zaragoza: 117-121.

Suárez MÁrouez, A. 1981: "Cueva de Ambrosio (Vélez-Blanco, Almeria). Nuevas aportaciones al estudio del Epipaleolítico del Sudeste Peninsular". Arqueología y Paleoecología Humana 2: 43-53. 